\title{
THERMOMECHANICAL SLIP IN ELASTIC CONTACT BETWEEN IDENTICAL MATERIALS
}

\author{
Yurii STRELIAIEV,$*+\infty$, Rostyslav MARTYNYAK ${ }^{*+*} \oplus$, Kostyantyn CHUMAK ${ }^{*+*} \oplus$ \\ *Faculty of Mathematics, Zaporizhzhia National University, 66, Zhukovsky street, Zaporizhzhia 69600, Ukraine \\ ${ }^{* *}$ Department of Natural Science, Zaporizhzhia Institute of Economics and Information Technologies, \\ 16b, Kyiashka street, Zaporizhzhia 69041, Ukraine \\ ${ }^{* * *}$ Department of Mathematical Problems of Contact Mechanics, Pidstryhach Institute for Applied Problems of Mechanics and Mathematics, \\ 3-b, Naukova street, Lviv 79060, Ukraine
}

strelkiny@gmail.com, m.rostyslav@gmail.com, chumakostya@gmail.com

received 8 November 2020, revised 19 July 2021, accepted 23 July 2021

\begin{abstract}
The contact problem for interaction between an elastic sphere and an elastic half-space is considered taking into account partial thermomechanical frictional slip induced by thermal expansion of the half-space. The elastic constants of the bodies are assumed to be identical. The Amontons-Coulomb law is used to account for friction. The problem is reduced to non-linear boundary integral equations that correspond to the initial stage of mechanical loading and the subsequent stage of thermal loading. The dependences of the contact stress distribution, relative displacements of the contacting surfaces, dimensions of the stick and slip zones on temperature of the half-space are studied numerically. It was revealed that an increase in temperature causes increases in the shear contact stress and the relative shear displacements of the contacting surfaces. The absolute values of the shear contact stress reach their maximum at the boundaries of the stick zones. The greatest value of the moduli of the relative shear displacements are reached at the boundary of the contact region. The stick zone radius decreases monotonically according to a nonlinear law with increasing temperature.
\end{abstract}

Key words: elastic contact, thermomechanical slip, stick and slip zones, contact stresses, integral equation, numerical solution, iterative method

\section{INTRODUCTION}

Solving contact problems on the interaction of elastic bodies often requires taking account of the friction between the contacting surfaces. The complexity of such problems is usually caused by the fact that the contact surface and the stick and slip zones arising on it are unknown and can have a complex unpredictable shape, which varies with the applied loading. These circumstances lead to nonlinearities in the formulation of such problems that significantly complicates their solving.

The investigations of partial frictional slip for various types of mechanical loading were initiated by Cattaneo (1938) and Mindlin (1949) and are actively continuing thus far. The reviews of such studies are presented in numerous papers (Kalker, 1977; Hills and Urriolagoitia Sosa, 1999; Barber and Ciavarella, 2000; Goryacheva and Martynyak, 2014) and books (Johnson, 1985; Hills et al., 1993; Ostryk and Ulitko, 2006; Popov, 2017; Barber, 2018; Ostryk, 2018).

In real operating conditions, the contacting bodies are often heated, and this can lead to change in the conditions in the contact region. Therefore, taking into account temperature effects plays an important role in the investigation of contact interactions. The major studies in this area are dedicated to investigation of the non-frictional thermoelastic contact (Borodachev, 1962; Grilitskii and Shelestovskii, 1970; Barber, 1973; Dundurs and Panek, 1976; Comninou et al., 1981; Krishtafovich and Martynyak, 1999; Kulchytsky-Zhyhailo et al., 2001; Martynyak and Chumak, 2012
Chumak 2018; Chumak and Martynyak, 2019) and investigation of the effects associated with heat generation due to sliding friction (Barber, 1976; Yevtushenko and Kulchytsky-Zhyhailo, 1996; Grilitskii and Pauk, 1997; Pauk, 2006; Kulchytsky-Zhyhailo et al., 2011).

However, thermal deformations can also cause partial slip of the contacting surfaces when bodies are heated. Determining distributions of the resultant shear contact stress as well as boundaries of slip and stick zones is especially important in the investigation of fretting wear and fatigue of real components of machines and structures (Hills and Urriolagoitia Sosa, 1999). Thermomechanical partial slip has been studied in a few works only. Pauk $(2005,2007)$ investigated thermally induced partial slip between a flat-ended punch and an elastic half-space with different temperatures. The thermoelastic stick-slip contact problem for two semi-infinite solids in the presence of a single thermoinsulated interface gap was studied by Malanchuk et al. (2011). The effect of thermal conductivity of an interstitial medium on partial slip between a textured half-space and a flat half-space, which is caused by an imposed heat flow, was examined by Chumak et al. (2014).

This paper aims to investigate partial frictional slip between the contacting surfaces of an elastic sphere and an elastic halfspace that is caused by sequentially applied mechanical and thermal loads. The effect of the thermal load on the shear contact stress, relative slip of the contacting surfaces, and dimensions of the stick and slip zones will be studied. 


\section{STATEMENT OF THE PROBLEM}

Consider the contact problem for an interaction between an elastic sphere and an elastic half-space. The bodies are assumed to be isotropic and have identical elastic properties characterised by Young's modulus E and Poisson's ratio $v$. The initial temperatures of the bodies are zero. The bodies are loaded in two stages. Initially the sphere is pressed into contact with the half-space by a normal force $\mathrm{P}$. The radius $\mathrm{a}_{0}$ of the resultant contact region is supposed to be small in comparison with the radius $\mathrm{R}$ of the sphere $\left(\mathrm{a}_{0} \ll \mathrm{R}\right)$. Then, while the normal force is held constant, the half-space is uniformly heated to the temperature $\mathrm{T}$ and the thermal linear expansion of the half-space occurs. The contact surface is assumed to be thermoinsulated. The relative displacements of the points of the contacting surfaces resulting from the thermal expansion of the half-space are partially restrained by friction obeying the Amontons-Coulomb law (Kalker, 1977).

We will consider the problem in a three-dimensional formulation. Let us introduce the coordinate system in such a way that the elastic half-space is determined by the inequality $\mathrm{z} \leq 0$, and the origin of the coordinate system is the point of initial contact between the sphere and the half-space (Fig. 1).

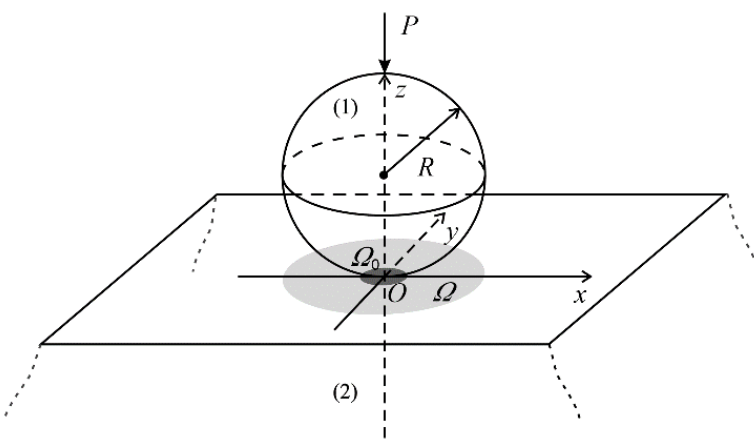

Fig. 1. The schema of contact interaction

Considering that the contact region radius is small in comparison with the sphere radius, the sphere can be replaced by the elastic half-space $\mathrm{z} \geq 0$. Below we will consider the contact boundary conditions separately for each of the two loading stages.

The boundary conditions at the first loading stage are specified in the bounded domain $\Omega$ of the plane $\mathrm{z}=0$, containing an unknown contact region $\Omega_{0}$. Since the contacting materials are identical, the elastic shear displacements of the points of the contacting surfaces are the same and friction does not arise (Johnson, 1985). Thus the shear stresses on the both contacting surfaces are zero and the boundary conditions in every point $\mathrm{s}=(\mathrm{x}, \mathrm{y}) \in \Omega$ can be written as follows (Barber, 2018):

$g(s) \geq 0, s \in \Omega$;

$p(s) \geq 0, s \in \Omega$;

$g(s) \cdot p(s)=0, s \in \Omega$.

In the relations expressed in Eq. (1), $g(s)$ is a function of a gap between the bodies:

$g(s)=u_{z}^{(1)}(s, 0)-u_{z}^{(2)}(s, 0)+g_{0}(s)-\Delta$,

where $\mathrm{u}_{\mathrm{z}}^{(1)}(\mathrm{s}, 0), \mathrm{u}_{\mathrm{z}}^{(2)}(\mathrm{s}, 0)$ denotes normal elastic displacements of the points of the contacting surfaces; $\Delta$ denotes the approach of two bodies; and $\mathrm{g}_{0}(\mathrm{~s})$ denotes the initial gap between the bodies in their undeformed state. As the sphere radius $\mathrm{R}$ is large in comparison with the contact region radius, the initial gap $g_{0}(s)$ near the origin of the coordinate system can be approximately represented in the following form (Johnson, 1985):

$g_{0}(s)=\frac{x^{2}+y^{2}}{2 R}$.

The function $p(s)$ in Eq. (1) describes the contribution of a contact pressure in the domain $\Omega$.

The condition (1a) means the non-negativity of the gap $g(s)$ between the bodies (there is no interpenetration), the condition (1b) means the non-negativity of the contact pressure $p(s)$, the condition (1c) means that contact pressure is zero outside the contact region and the gap is zero inside this region.

The relationship between the unknown functions $g(s)$ and $p(s)$, in accordance with the Boussinesq's solution (Johnson, 1985), is expressed in the following integral form

$g(s)=2 \cdot \frac{\left(1-v^{2}\right)}{\pi E} \int_{\Omega} \frac{p\left(s^{\prime}\right)}{r} d s^{\prime}+g_{0}(s)-\Delta$,

where $r=\left|s-s^{\prime}\right|=\sqrt{\left(x-x^{\prime}\right)^{2}+\left(y-y^{\prime}\right)^{2}}$.

The relations obtained in Eq. (1) should be supplemented with the following equilibrium condition:

$P=\int_{\Omega} p(s) d s$.

When the approach $\Delta$ of the bodies is given, the condition in Eq. (5) is used for determination of the pressing force $P$ ensuring the approach $\Delta$.

Thus, at the first loading stage, the problem is reduced to determination of the function $p(s)$, which satisfies the boundary conditions in Eq. (1) (taking into account the relations in Eq. (4)) at every point of the domain $\Omega$. Once the contact pressure $p(s)$ is found, the contact region $\Omega_{0}$ is determined from the condition $g(s)=0$.

Heating the lower half-space to the constant temperature $\mathrm{T}>0$ gives rise to thermal shear displacements of its boundary. These displacements lead to the appearance of a peripheral annular zone of slippage of the contacting surfaces, where friction forces act, and a central circular zone $\Omega_{1}$ of stick of the contacting surfaces. The radius of the zone $\Omega_{1}$ is $a_{1}\left(a_{1}<a_{0}\right)$.

Therefore, the thermal expansion of the surface of the lower half-space in the presence of friction will cause relative shear displacements of the contacting surfaces $u_{x}(s), u_{y}(s)$ and the shear stresses $\tau_{z x}^{(1)}(s, 0), \tau_{z y}^{(1)}(s, 0), \tau_{z x}^{(2)}(s, 0) \tau_{z y}^{(2)}(s, 0)$ on the contacting surfaces.

When the elastic constants of the bodies are the same, the shear stresses do not affect the geometry of the contact region $\Omega_{0}$ and the distribution of the contact pressures $p(s)$ (Johnson, 1985). Thus, we will assume that the domain $\Omega_{0}$ as well as the function $p(s)$ are known and have been determined when solving the problem for the first loading stage.

The following formulas are valid for displacements $u_{x}(s)$ and $u_{y}(s)$ :

$u_{x}(s)=u_{x}^{(1)}(s, 0)-u_{x}^{(2)}(s, 0)-\tilde{u}_{x}^{(2)}(s)$,

$u_{y}(s)=u_{y}^{(1)}(s, 0)-u_{y}^{(2)}(s, 0)-\tilde{u}_{y}^{(2)}(s)$,

where: $u_{x}^{(1)}(s, 0), u_{y}^{(1)}(s, 0)$ and $u_{x}^{(2)}(s, 0), u_{y}^{(2)}(s, 0)$ are the elastic shear displacements of the upper and the lower contacting 
surfaces that are caused by the contact shear stresses; $\tilde{u}_{x}^{(2)}(s)=\alpha \cdot x \cdot \mathrm{T}, \quad \tilde{u}_{y}^{(2)}(s)=\alpha \cdot y \cdot \mathrm{T} \quad$ are the shear displacements of the points of the lower contacting surface that are caused by thermal expansion of the lower half-space ( $\alpha$ denotes the coefficient of linear thermal expansion) (Nowacki, 1986).

The contact boundary conditions for the second loading stage include the conditions of equality of shear stresses on the surfaces inside the contact region $\Omega_{0}$

$\tau_{z x}^{(1)}(s, 0)=\tau_{z x}^{(2)}(s, 0), \tau_{z y}^{(1)}(s, 0)=\tau_{z y}^{(2)}(s, 0), s \in \Omega_{0}$

and the relations of the Amontons-Coulomb law.

In view of Eq. (7), it is sufficient to write the AmontonsCoulomb law for the contact stress acting on the upper contacting surface only.

Denoting

$q_{x}(s) \equiv-\tau_{z x}^{(1)}(s, 0), q_{y}(s) \equiv-\tau_{z y}^{(1)}(s, 0)$,

the relations of the Amontons-Coulomb law can be represented as (Kalker, 1977)

$|\vec{q}| \leq \mu \cdot p(s), s \in \Omega_{0}$,

$|\vec{u}| \neq 0 \Rightarrow \vec{q}=-\mu \cdot p(s) \cdot \frac{\vec{u}}{|\vec{u}|}, s \in \Omega_{0}$,

where: $\vec{q}=\left(q_{x}(s), q_{y}(s)\right)$ is the vector of specific shear stress acting on the upper contacting surface at the point $s$; $\vec{u}=\left(u_{x}(s), u_{y}(s)\right)$ is the vector of shear displacement of the upper contacting surface with respect to the lower one at the point $s$; and $\mu$ denotes the coefficient of friction.

Passing from the vectors $\vec{q}, \vec{u}$ to their components, the conditions in Eq. (9) can be rewritten in the following equivalent form:

$\sqrt{q_{x}^{2}(s)+q_{y}^{2}(s)} \leq \mu \cdot p(s), s \in \Omega_{0} ;$

$q_{x}(s) \sqrt{u_{x}^{2}(s)+u_{y}^{2}(s)}+\mu \cdot p(s) u_{x}(s)=0, s \in \Omega_{0} ;$

$q_{y}(s) \sqrt{u_{x}^{2}(s)+u_{y}^{2}(s)}+\mu \cdot p(s) u_{y}(s)=0, s \in \Omega_{0}$.

The condition (10a) means that the absolute value of the shear stress at any point of the contact region $\Omega_{0}$ does not exceed the product of the coefficient of friction $\mu$ and the contact pressure value at this point. The conditions (10b) and (10c) mean that for all the points of the contact region where slippage takes place $(|\vec{u}| \neq 0)$ the equality $|\vec{q}|=\mu \cdot p(s)$ holds, and the vector of shear stress $\vec{q}$ and vector of the relative displacement of the contacting surfaces $\vec{u}$ have opposite direction. For stick $(|\vec{u}|=0)$, the equalities (10b) and (10c) become identities.

According to the Cerruti solution (Johnson, 1985), the differences in the shear elastic displacements in Eq. (6) can be expressed in the following integral form:

$u_{x}^{(1)}(s, 0)-u_{x}^{(2)}(s, 0)=\int_{\Omega} K_{x x}\left(s, s^{\prime}\right) q_{x}\left(s^{\prime}\right) d s^{\prime}+$ $\int_{\Omega} K_{x y}\left(s, s^{\prime}\right) q_{y}\left(s^{\prime}\right) d s^{\prime}$,

$u_{y}^{(1)}(s, 0)-u_{y}^{(2)}(s, 0)=\int_{\Omega} K_{y x}\left(s, s^{\prime}\right) q_{x}\left(s^{\prime}\right) d s^{\prime}+$ $\int_{\Omega} K_{y y}\left(s, s^{\prime}\right) q_{y}\left(s^{\prime}\right) d s^{\prime}$,

where:

$$
\begin{aligned}
& K_{x x}\left(s, s^{\prime}\right)=2 \cdot \frac{\left(1-v^{2}\right)}{\pi E} \cdot \frac{1}{r}+2 \cdot \frac{v(1+v)}{\pi E} \cdot \frac{\left(x-x^{\prime}\right)^{2}}{r^{3}} ; \\
& K_{x y}\left(s, s^{\prime}\right)=K_{y x}\left(s, s^{\prime}\right)=2 \cdot \frac{v(1+v)}{\pi E} \cdot \frac{\left(x-x^{\prime}\right)\left(y-y^{\prime}\right)}{r^{3}} ; \\
& K_{y y}\left(s, s^{\prime}\right)=2 \cdot \frac{\left(1-v^{2}\right)}{\pi E} \cdot \frac{1}{r}+2 \cdot \frac{v(1+v)}{\pi E} \cdot \frac{\left(y-y^{\prime}\right)^{2}}{r^{3}} ; \\
& r=\left|s-s^{\prime}\right|=\sqrt{\left(x-x^{\prime}\right)^{2}+\left(y-y^{\prime}\right)^{2}} .
\end{aligned}
$$

Therefore, the problem for the second loading stage is reduced to determining the functions $q_{x}(s), q_{y}(s)$, which satisfy the relations obtained in Eq. (10) (taking into account Eqs (6) and (11)) at every point of the contact region $\Omega_{0}$, and the function $p(s)$ is known from the solution of the problem for the first loading stage. Once the distributions of the shear contact stresses $q_{x}(s), q_{y}(s)$ are found, the stick zone $\Omega_{1}$ is determined from the condition $|\vec{u}|=0$.

\section{INTEGRAL EQUATIONS OF THE CONTACT PROBLEM}

To obtain integral equations describing the contact interaction of the bodies at the first and second loading stages, we use the approach proposed in the literature (Aleksandrov, 2015; Alexandrov and Streliaiev, 2014; Streliaiev, 2016).

According to it, consider the helper functions:

$H(x)=\left\{\begin{array}{l}x, \text { if } x \geq 0, \\ 0, \text { if } x<0,\end{array}\right.$

$Q(x, y, z)= \begin{cases}x, & \text { if } \sqrt{x^{2}+y^{2}} \leq z, \\ \frac{x z}{\sqrt{x^{2}+y^{2}}}, \text { if } \sqrt{x^{2}+y^{2}}>z .\end{cases}$

The following theorems (provided below without proof) hold for the properties of the helper functions (Aleksandrov, 2015).

Theorem 1. For any real numbers $x, y$, the system

$\left\{\begin{array}{l}x \geq 0 \\ y \geq 0 \\ x \cdot y=0\end{array}\right.$

is equivalent to the equality

$x=H(x-E \cdot y)$,

where $E$ is an arbitrary positive number.

Theorem 2. For any real numbers $x, y, u, v$ and any nonnegative number $z$, the system of relations

$\left\{\begin{array}{l}\sqrt{x^{2}+y^{2}} \leq z, \\ x \cdot \sqrt{u^{2}+v^{2}}+z \cdot u=0, \\ y \cdot \sqrt{u^{2}+v^{2}}+z \cdot v=0\end{array}\right.$

is equivalent to the system of equalities

$\left\{\begin{array}{l}x=Q(x-E u, y-E v, z), \\ y=Q(y-E v, x-E u, z),\end{array}\right.$

where $E$ is an arbitrary positive number.

As follows from the Theorem 1, boundary conditions in Eq. (1) with the use of the function $H$ can be expressed as a single nonlinear integral equation for the function $p(s)$ defined in the domain $\Omega$ : 
$p(s)=H\left(p(s)-\mathrm{E}_{1} \cdot g(s)\right)$

where $\mathrm{E}_{1}$ is an arbitrary positive number.

In view of the Theorem 2, the boundary conditions in Eq. (10) are reduced to a system of nonlinear integral equations for the functions $q_{x}(s), q_{y}(s)$ defined in the domain $\Omega_{0}$ :

$\left\{\begin{array}{l}q_{x}(s)=Q\left(q_{x}(s)-\mathrm{E}_{2} u_{x}(s), q_{y}(s)-\mathrm{E}_{2} u_{y}(s), \mu \cdot p(s)\right), \\ q_{y}(s)=Q\left(q_{y}(s)-\mathrm{E}_{2} u_{y}(s), q_{x}(s)-\mathrm{E}_{2} u_{x}(s), \mu \cdot p(s)\right),\end{array}\right.$

where $E_{2}$ is an arbitrary positive number, and the non-negative function $p(s)$ is known from the solution of Eq. (18).

The method proposed by Aleksandrov (2015) is utilised to solve the system of integral Eqs (18) and (19) numerically. This method includes regularisation of integral equations, discretisation of the regularised equations and usage of an iterative process for obtaining an approximate solution to discrete analogs of the regularised equations.

Such discrete analogs can be obtained by defining the domain $\Omega$ as an open square which is bounded by straight lines parallel to the axes $O x, O y$. Let us divide the domain $\Omega$ for every positive integer $n$ into $n^{2}$ square domains $\omega_{1}, \omega_{2}, \ldots, \omega_{n^{2}}$ of equal area that are arranged as the square $\Omega$. Assuming that the unknown function $p(s)$ has a constant value $p_{k}$ on every element $\omega_{k}$ at the first loading stage, we obtain the following system of $n^{2}$ nonlinear scalar equations for an approximate solution to Eq. (18):

$p_{k}=H\left(p_{k}-\mathrm{E}_{1} \cdot\left(\sum_{j=1}^{n^{2}} a_{k j}^{1} \cdot p_{j}-b_{k}^{1}\right)\right)$.

We assume that the functions $q_{x}(s)$ and $q_{y}(s)$ have constant values $q_{2 k-1}$ and $q_{2 k}$ on every element $\omega_{k}$ at the second loading stage. Then the discrete analog of the system in Eq. (19) has the following form:

$$
\left\{\begin{array}{c}
q_{2 k-1}=Q\left(q_{2 k-1}-\mathrm{E}_{2} \cdot\left(\sum_{j=1}^{2 n^{2}} a_{2 k-1 j}^{2} \cdot q_{j}-b_{2 k-1}^{2}\right),\right. \\
\left.q_{2 k}-\mathrm{E}_{2} \cdot\left(\sum_{j=1}^{2 n^{2}} a_{2 k j}^{2} \cdot q_{j}-b_{2 k}^{2}\right), \mu \cdot p_{k}\right) ; \\
q_{2 k}=Q\left(q_{2 k}-\mathrm{E}_{2} \cdot\left(\sum_{j=1}^{2 n^{2}} a_{2 k j}^{2} \cdot q_{j}-b_{2 k}^{2}\right),\right. \\
\left.q_{2 k-1}-\mathrm{E}_{2} \cdot\left(\sum_{j=1}^{2 n^{2}} a_{2 k-1 j}^{2} \cdot q_{j}-b_{2 k-1}^{2}\right), \mu \cdot p_{k}\right) .
\end{array}\right.
$$

In (20) and (21), $k=\overline{1, n^{2}}$, numerical parameters $a_{i j}^{1}$ and $a_{k j}^{2}$ are the elements of the compliance matrix of the contacting bodies, $b_{i}^{1}$ and $b_{i}^{2}$ define the loading conditions at the first and second stages respectively. The approximate solutions of the systems in Eqs (20) and (21) are found using the iterative method (Aleksandrov, 2015).

\section{NUMERICAL RESULTS AND THEIR ANALYSIS}

Numerical calculations are performed for the coefficient of friction $\mu=0.5$, Poisson's ratio $v=0.3$ and the dimensionless quantities

$\bar{x}=\frac{x}{R}, \quad \bar{y}=\frac{y}{R}, \quad \bar{p}(s)=\frac{p(s)}{E}, \quad \bar{q}_{x}(s)=\frac{q_{x}(s)}{E}$,

$\bar{q}_{y}(s)=\frac{q_{y}(s)}{E}, \quad \bar{u}_{x}(s)=\frac{u_{x}(s)}{R}, \quad \bar{u}_{y}(s)=\frac{u_{y}(s)}{R}$,

$\bar{P}=\frac{P}{E \cdot R^{2}}, \quad \bar{\Delta}=\frac{\Delta}{R}, \quad \bar{a}_{1}=\frac{a_{1}}{R}, \quad \overline{\mathrm{T}}=\alpha \cdot \mathrm{T}$.
Fig. 2 shows the contact pressure $\bar{p}=\bar{p}(\bar{x}, 0)$ versus the coordinate $\bar{x}$ when the approach of the bodies equals $\bar{\Delta}=2.7$. $10^{-4}$ and the corresponding pressing force is $\bar{P}=3.2 \cdot 10^{-6}$. The solid line corresponds to the Hertz pressure distribution for an axisymmetric normal contact (Johnson, 1985), and the circles correspond to the numerical solution of Eq. (20) (the first loading stage, $\bar{T}=0$ ).

As seen in Fig. 2, the numerical solution of the problem for the first loading stage and the Hertz analytical solution agree very closely.

To analyse the effect of thermomechanical slip on the contact interaction parameters, numerical calculations are performed for various values of temperature $\overline{\mathrm{T}}$ at the fixed value of the pressing force $\bar{P}=3.2 \cdot 10^{-6}$.

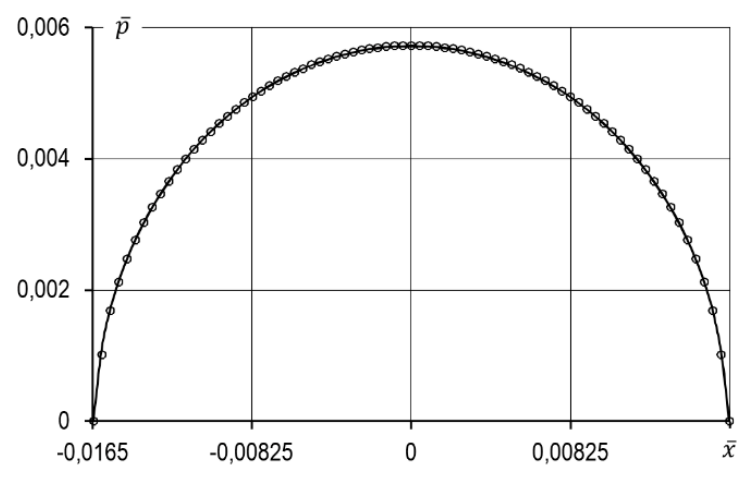

Fig. 2. Contact pressure distribution

Fig. 3 shows the shear contact stress $\bar{q}_{x}=\bar{q}_{x}(x, 0)$ versus the coordinate $\bar{x}$ for various values of temperature $\bar{T}$. The curves have kinks at the points $\bar{x}= \pm \bar{a}_{1}$ corresponding to the ends of stick zones. As temperature is increased, the absolute value of the shear contact stress $\left|\overline{\mathrm{q}}_{\mathrm{x}}(\mathrm{x}, 0)\right|$ increases and reaches its maximum at the ends of stick zones. The curve $\left|\overline{\mathrm{q}}_{\mathrm{x}}(\mathrm{x}, 0)\right|$ within the slip zones coincides with the curve $\mu \cdot \overline{\mathrm{p}}(\overline{\mathrm{x}}, 0)$, therefore the shear stress is zero at the ends of the contact region. Within the stick zone, the shear stress is less than pressure multiplied by the coefficient of friction and is zero in the center of the contact region (at $\overline{\mathrm{x}}=0$ ).

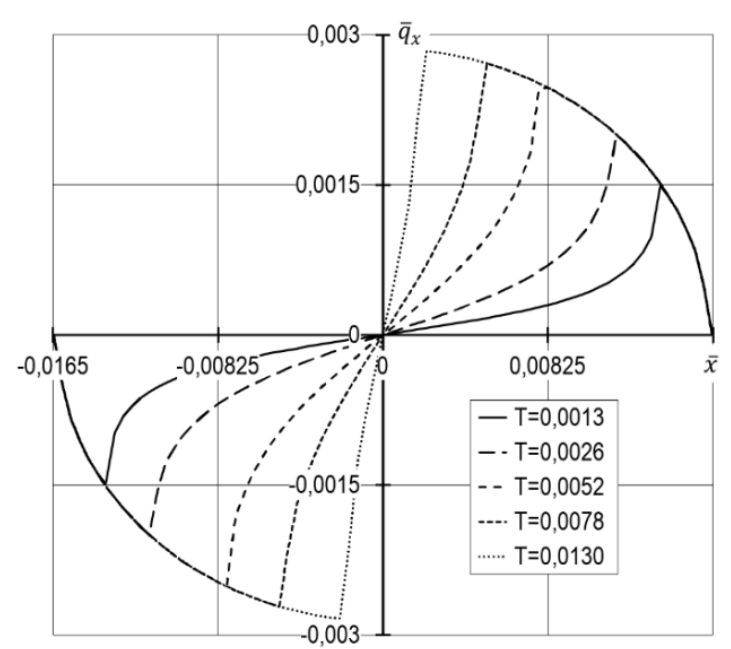

Fig. 3. Shear contact stress distribution 
Fig. 4 shows the relative shear displacements $\bar{u}_{x}=$ $\bar{u}_{x}(x, 0)$ of the contacting surfaces in the contact region versus the coordinate $\bar{x}$ for various values of temperature $\bar{T}$. The horizontal sections lying on the axis $O \bar{x}$ correspond to the stick zones, and the curved sections correspond to the slip zones where the relative shear displacements of the surfaces occur. The relative shear displacements increase with increasing temperature, and their maximum value is reached at the boundary of the contact region.

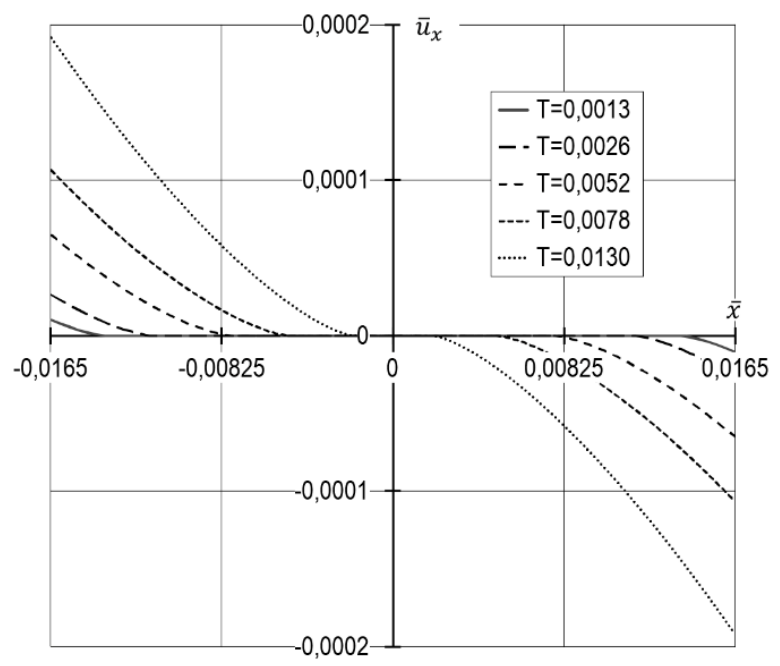

Fig. 4. Relative shear displacements of contacting surfaces versus coordinate

Fig. 5 shows the approximate boundaries of the stick zones calculated numerically for different values of temperature. Here, $\overline{\mathrm{T}}=0$ corresponds to the contact region at the first loading stage. The contact region does not change at the second stage of thermal loading. The perimeters of the regions with different shades of gray correspond to boundaries of the circular stick zones and annular slip zones which arise at different discrete values of the heating temperature $\overline{\mathrm{T}}$ of the lower half-space (from $\overline{\mathrm{T}}=0.0013$ to $\overline{\mathrm{T}}=0.013$ ).

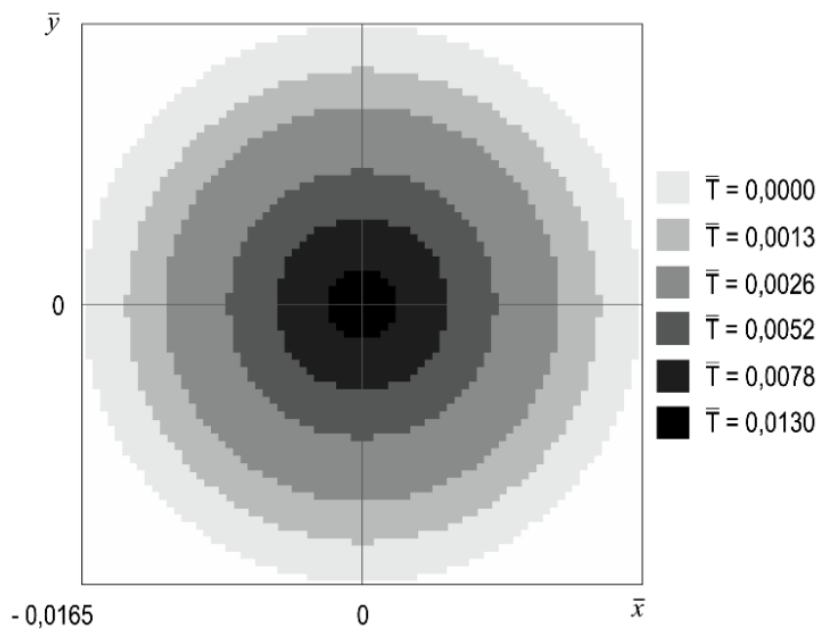

Fig. 5. Shape of stick zones

Fig. 6 shows the stick zone radius $\bar{a}_{1}$ versus temperature $\overline{\mathrm{T}}$. The circles correspond to the numerical results and the solid line corresponds to the fitting curve. The stick zone radius nonlinearly depends on temperature and decreases monotonically with temperature increasing. The obtained curve suggests that the stick zone radius will asymptotically tend to zero as the temperature tends to infinity, and the stick region itself will contract to a point.

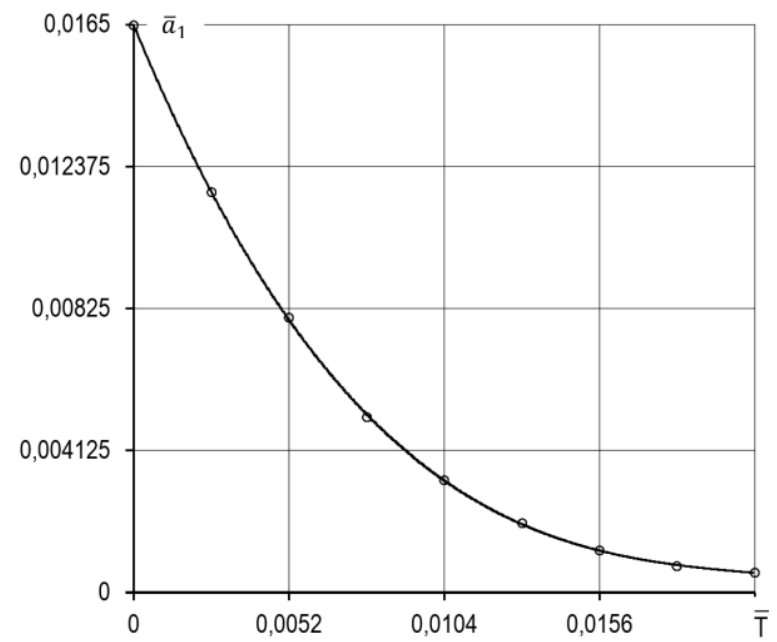

Fig. 6. Stick zone radius versus temperature

\section{CONCLUSIONS}

The problem for contact interaction between identically elastic sphere and half-space has been formulated taking into account partial thermomechanical frictional slip, which arises in the contact region due to thermal expansion of the half-space.

Nonlinear boundary integral equations that describe the contact interaction of the bodies at successive stages of mechanical and thermal loading were obtained. To solve the equations numerically, the method proposed by Aleksandrov (2015) was utilised. It includes regularisation of the integral equations, discretisation of the regularised equations and the use of an iterative process to obtain an approximate solution of discrete analogs of the regularised equations.

The effect of temperature of the half-space on the distribution of contact stresses, the relative displacements of the contacting surfaces, the dimensions of the stick and slip zones was studied. It was revealed that an increase in temperature causes increases in the shear contact stress and the relative shear displacements of the contacting surfaces. The absolute values of the shear contact stress reach their maximum at the boundaries of the stick zones. The greatest value of the moduli of the relative shear displacements are reached at the boundary of the contact region. The stick zone radius decreases monotonically according to a nonlinear law with increasing temperature.

The obtained results can be useful for analysing different types of failure, such as fretting fatigue, creep failure and crack nucleation.

\section{REFERENCES}

1. Aleksandrov A.I. (2015), A Method for the Solution of a ThreeDimensional Contact Problem of Interaction of Two Elastic Bodies in the Presence of Friction, Journal of Mathematical Sciences, 205(7), 518-534. 
2. Alexandrov A., Streliaiev Y. (2014), Nonlinear boundary integral equation's method for elastic contact problems, Eastern-European Journal of Enterprise Technologies, 3(7), 36-40 (in Russian).

3. Barber J.R. (1973), Indentation of a semi-infinite elastic body by a hot sphere, International Journal of Mechanical Sciences, 15(10), 813-819.

4. Barber J.R. (1976), Some thermoelastic contact problems involving frictional heating, The Quarterly Journal of Mechanics and Applied Mathematics, 29(1), 1-13.

5. Barber J.R. (2018), Contact mechanics. Solid Mechanics and lts Applications, Springer.

6. Barber J.R., Ciavarella M. (2000), Contact mechanics, International Journal of Solids and Structures, 37(1-2), 29-43.

7. Borodachev N.M. (1962), On solution of contact problem of thermoelasticity in a case of axial symmetry, Izv. Akad. Nauk SSSR, Techn. Mekh. Mashinstr., 5, 86-90 (in Russian).

8. Cattaneo C. (1938), Sul contatto di due corpi elastici: distribuzione locale degli stozzi, Rend. Dell'Academia nazionale dei Lincei, 27(6), 342-348, 434-436, 474-478

9. Chumak K. (2018), The thermoelastic contact problem for wavy surfaces with a heat-conducting medium in interface gaps, Mathematics and Mechanics of Solids, 23(10), 1389-1406.

10. Chumak K., Malanchuk N., Martynyak R. (2014), Partial slip contact problem for solids with regular surface texture assuming thermal insulation or thermal permeability of interface gaps, International Journal of Mechanical Sciences, 84, 138-146.

11. Chumak K., Martynyak R. (2019), The combined thermal and mechanical effect of an interstitial gas on thermal rectification between periodically grooved surfaces, Front Mech Eng, 5(42), 1-8.

12. Comninou M., Dundurs J., Barber J.R. (1981), Planar Hertz contact with heat conduction, Journal of Applied Mechanics, 48(3), 549-554.

13. Dundurs J., Panek C. (1976), Heat conduction between bodies with wavy surfaces, International Journal of Heat and Mass Transfer 19(7), 731-736

14. Goryacheva I.G., Martynyak R.M. (2014), Contact problems for textured surfaces involving frictional effects, Proceedings of the Institution of Mechanical Engineers, Part J: Journal of Engineering Tribology, 228(7), 707-716.

15. Grilitskii D.V., Pauk V.I. (1997), The plane contact problem of steady thermoelasticity taking heat generation into account, Journal of Applied Mathematics and Mechanics, 61(6), 1007-1012.

16. Grilitskii D.V., Shelestovskii B.G. (1970), The axisymmetric contact problem of thermoelasticity for a transversely isotropic half-space, Soviet Applied Mechanics, 6(8), 807-811.

17. Hills D.A., Nowell D., Sackfield A. (1993), Mechanics of Elastic Contact, Butterworth-Heinemann, Oxford.

18. Hills D.A., Urriolagoitia Sosa G. (1999), Origins of partial slip in fretting - a review of known and potential solutions, The Journal of Strain Analysis for Engineering Design, 34(3), 175-181.

19. Johnson K.L. (1985), Contact mechanics, Cambridge University Press, Cambridge.

20. Kalker J.J. (1977), A survey of the mechanics of contact between solid bodies, ZAMM, 57(5), T3-T17.

21. Krishtafovich A.A., Martynyak R.M. (1999), Lamination of anisotropic half-spaces in the presence of contact thermal resistance International Applied Mechanics, 35(2),159-164.

22. Kulchytsky-Zhyhailo R.D., Matysiak S.J., Perkowski D.M. (2011), Plane contact problems with frictional heating for a vertically layered half-space, International Journal of Heat and Mass Transfer, 54(9), 1805-1813.

23. Kulchytsky-Zhyhailo R.D., Olesiak Z.S., Yevtushenko 0.0. (2001), On thermal contact of two axially symmetric elastic solids, Journal of Elasticity, 63(1), 1-17.

24. Malanchuk N., Martynyak R., Monastyrskyy B. (2011), Thermally induced local slip of contacting solids in vicinity of surface groove, International Journal of Solids and Structures, 48(11-12), 17911797.
25. Martynyak R., Chumak K. (2012), Effect of heat-conductive filler of interface gap on thermoelastic contact of solids, International Journal of Heat and Mass Transfer, 55(4), 1170-1178.

26. Mindlin R.D. (1949), Compliance of elastic bodies in contact, Trans. ASME, J. Appl. Mech., 16(3), 259-268.

27. Nowacki W. (1986), Thermoelasticity. 2nd edn., PWN-Polish Scientific Publishers.

28. Ostryk V.I. (2018), Factorization method and its generalization in mixed problems of elasticity theory, VPC «Kyivskyi universytet», Kyiv (in Ukrainian)

29. Ostryk V.I., Ulitko A.F. (2006), The Wiener-Hopf Method in Contact Problems of Elasticity Theory, Naukova Dumka, Kyiv (in Ukrainian).

30. Pauk V. (2006), Plane contact problems involving frictional heating for wavy half-space, Tribology Letters, 24(6), 237-242.

31. Pauk V. (2007), Plane contact of hot flat-ended punch and thermoelastic half-space involving finite friction, Journal of Applied Mechanics, 74(6), 1172-1177.

32. Pauk W. (2005), Wybranie zagadnienia kontaktu cial odksztalcalnych, Kielce: Wydawnictwo Politechniki Swietokrzyskiej (in Polish).

33. Popov V.L. (2017), Contact Mechanics and Friction. Physical Principles and Applications, Springer, Berlin.

34. Streliaiev Yu. M. (2016). A nonlinear boundary integral equations method for the solving of quasistatic elastic contact problem with Coulomb friction, Journal of Samara State Technical University, Ser. Physical and Mathematical Sciences, 20(2), 306-327 (in Russian).

35. Yevtushenko A.A., Kulchytsky-Zhyhailo R.D. (1996) Approximation solution of the thermoelastic contact problem with frictional heating in the general case of the profile shape, Journal of the Mechanics and Physics of Solids, 44, 243-250.

Yurii Streliaiev: (iD) https://orcid.org/0000-0002-4400-7824

Rostyslav Martynyak: (D) https://orcid.org/0000-0001-7613-1427

Kostyantyn Chumak: (iD https://orcid.org/0000-0002-7033-0836 